\title{
A 3D hybrid simulation study of the electromagnetic field distributions in the lunar wake
}

\author{
Y.-C. Wang ${ }^{\mathrm{a}, *}$, J. Müller ${ }^{\mathrm{b}}$, W.-H. Ip ${ }^{\mathrm{a}, \mathrm{c}}$, U. Motschmann ${ }^{\mathrm{b}, \mathrm{d}}$ \\ a Institute of Astronomy, National Central University, No. 300, Jhongda Rd., Jhongli City, Taoyuan County 32001, Taiwan, ROC \\ ' Institut für Theoretische Physik, Technische Universität Braunschweig, Mendelssohnstrasse 3, 38106 Braunschweig, Germany \\ ${ }^{\mathrm{c}}$ Institute of Space Science, National Central University, No. 300, Jhongda Rd., Jhongli City, Taoyuan County 32001, Taiwan, ROC \\ d Institut für Planetenforschung, DLR, German Aerospace Center, Rutherfordstrasse 2, 12489 Berlin, Germany
}

\section{A R T I C L E I N F O}

\section{Article history:}

Received 21 December 2010

Revised 25 August 2011

Accepted 21 September 2011

Available online 29 September 2011

\section{Keywords:}

Moon

Solar wind

Magnetic fields

\begin{abstract}
A B S T R A C T
A three-dimensional hybrid code is used to study the electromagnetic disturbances in the solar wind that arise due to the absorption effect of the Moon. Due to the nearly insulating nature of the Moon, interplanetary magnetic fields (IMFs) can move through the interior without hindrance. However, the near-vacuum created in the wake region due to the lunar absorption effect will lead to enhancement of the strength of the magnetic field by a factor of about 1.4 in the middle of the lunar wake and lead to depletions at two sides. The situations arising from different orientations of the interplanetary magnetic fields relative to the radial direction are compared. Asymmetries of the inward diffusions both along and perpendicular to the field lines are also observed. The electric field formed from the plasma convection could reach a magnitude of $0.2-0.8 \mathrm{mV} / \mathrm{m}$ at the border of the wake. The role of the electric field on the inward accelerations is important to the geometry of the lunar wake.
\end{abstract}

(c) 2011 Elsevier Inc. All rights reserved.

\section{Introduction}

As a consequence of its lack of a thick atmosphere and an ionosphere, the interaction of the solar wind with the Moon is characterized by the direct impact of the solar wind on its sunward hemisphere. This absorption effect produces a near-vacuum in the wake immediately behind the Moon. The absence of a global magnetosphere and the low electrical conductivity further leads to the free passage of the interplanetary magnetic field (IMF) through the lunar interior. This classic scenario of the solar wind-Moon interaction was established by the very first plasma measurements in the lunar environment made by the Explorer 35 spacecraft (Lyon et al., 1967; Schubert and Lichtenstein, 1974). The wake region is gradually filled in by the diffusion of solar wind protons into the zone of density depletion. As described in the early analyses by Michel (1968) and Whang and Ness (1970), the expansion of the solar wind plasma into the wake is accompanied by rarefaction waves. At the same time, the magnetometer experiment on Explorer 35 detected the existence of a field reduction zone at the wake boundary surrounding the central region, with magnetic field enhancement up to a factor of 1.4 in comparison to the value in the ambient solar wind (Colburn et al., 1967). Some of these features were repeatedly observed at different distances in the lunar wake by the Wind

\footnotetext{
* Corresponding author.

E-mail address: m949001@astro.ncu.edu.tw (Y.-C. Wang).
}

spacecraft (Ogilvie et al., 1996; Owen et al., 1996) and the Lunar Prospector (Halekas et al., 2005). Owen et al. (1996) pointed out that the patterns of magnetic field variations in the lunar wake can be explained in terms of a diamagnetic current system maintained by the density gradient at the wake boundary. The pressure balance condition in the plasma void would lead to a buildup of magnetic field pressure of the observed magnitude (Michel, 1968). In addition to the observations of plasma expansion in the lunar wake, as discussed by Samir et al. (1983), Ogilvie et al. (1996) detected the presence of cold ion beams in the density cavity from the Wind spacecraft measurements at a crossing distance of 6.5 lunar radii $\left(R_{L}=1737 \mathrm{~km}\right)$. If the ion beams are related to the acceleration by an electrostatic electric field near the wake boundary, then the strength of the electric field can be estimated to be about $2 \times 10^{-4} \mathrm{~V} / \mathrm{m}$.

In theoretical studies, Farrell et al. $(1998,2008)$ and Birch and Chapman $(2001,2002)$ used 1D and 2D electromagnetic "particlein-cell" codes to address the issue of the formation of a radially inward pointing ambipolar electric field, because of the fast refilling of the lunar wake by electrons. These authors demonstrated that the dynamics of the ions (and electrons also) are governed by electric fields created by the fast-moving electrons. Electron twostream instability can lead to the trapping of electrons in center of the wake. Furthermore, the refilling/diffusion process is predominantly aligned with the IMF direction. Using a two-dimensional hybrid code, Trávníček et al. (2005) studied the generation and nature of low-frequency turbulence in the lunar wake. Kallio 
(2005) carried out the first 3D hybrid computation of the solar wind-Moon interaction. His global simulations allowed for a comparison of the plasma and magnetic field variations along the lunar wake. The anisotropic compression and enhancement of the magnetic field in the central region of the lunar wake were reproduced in this work. Due to the fact that the electrons mainly act as a charge-neutralizing fluid, no ambipolar electric field created by charge separation is possible in the hybrid simulations. However, both Trávníček et al. (2005) and Kallio (2005) showed that the reduction of the electron pressure at the wake boundary could lead to an electric field $E_{r}\left(\sim-\nabla P_{e} / n e\right.$, where $n$ is the solar wind plasma density and $e$ is the electronic charge), of similar magnitude to the electrostatic field deduced by the Wind spacecraft measurements (Ogilvie et al., 1996). Recently, more detailed 3D hybrid simulation comparisons with the Wind spacecraft measurements have also been made by Holmström et al. (2011).

Clack et al. (2004) examined the proton velocity distributions measured by the Wind spacecraft at large downstream distances $\left(\sim 15-25 R_{L}\right)$. They found large ion temperature anisotropies in the lunar wake. This effect is consistent with the double-adiabatic invariants expected in the plasma expansion process, i.e., $T_{\perp} / B=$ constant and $T_{\|}(B / n)^{2}=$ constant. More recently, the plasma measurements of the Kaguya lunar orbiter have shown that a small fraction $(\sim 0.1-1 \%)$ of the solar wind protons could be reflected after surface impact and re-enter the lunar wake by means of their large gyroradii. This effect is defined as 'Type-II entry' by Saito et al. (2008) and Nishino et al. (2009a). Holmström et al. (2010) performed particle simulations to study the solar wind proton reflection effects. It is expected that new measurements to be made by the Artemis spacecraft in the lunar environment will provide more detailed information on the plasma wake effects for in-depth theoretical investigations and comparison (Halekas et al., 2011; Wiehle et al., 2011). In addition to the Artemis observations, it is expected that the upcoming encounter of the NASA's Dawn spacecraft with the second largest asteroid, Vesta, in July, 2011, will trigger further interest in the problems of solar wind-asteroid interactions (Simon et al., 2006a). Similar reasoning can be applied to the topic of stellar wind-(non-conducting) exoplanet interaction as considered by Lipatov et al. (2005). In the wake of these new developments, a 3D hybrid code is used to explore the global nature of solar wind plasma dynamics in the lunar wake, especially on the asymmetric features.

The rest of the paper is organized as follows. The basic scheme of the theoretical model is described in Section 2. The numerical setting and the effects of the lunar conductivity are discussed in Section 3. The details of the wake structures are addressed in Sections 4 and 5. The 'Type-I' and 'Type-II entry' of the solar wind protons in the near wake region, as reported in Saito et al. (2008) and Nishino et al. (2009a,b) are discussed in Section 6. A short summary and conclusions are given in Section 7.

\section{Model initialization}

The hybrid model used in this work is based on the 3D code developed by Bagdonat and Motschmann (2002) and Bagdonat (2004). It has been successfully applied for the simulation of various solar wind or magnetospheric interactions of planetary bodies (Bagdonat and Motschmann, 2002; Bößwetter et al., 2004; Simon et al., 2006a; Simon et al., 2006b; Roussos et al., 2008; Kriegel et al., 2009; Wang et al., 2010). Of special interest in the present context is Rhea's magnetospheric interaction, which has been investigated by Roussos et al. (2008). Similar to the case for the Moon, Rhea is also an insulator that can absorb the incoming plasma without hindrance. The main difference is that the Moon is interacting with a supersonic plasma flow rather than the subsonic interaction of Rhea, thus allowing the formation of a long wake with low density.

While the ions are treated as particles in the hybrid model, the electrons are taken as a neutralizing fluid. Thus, no charge separation can be considered in hybrid simulations. The full set of equations describing the motion of the solar wind protons and the charge-neutralizing electron components are given below. The equations for the ion motions are

$$
\begin{aligned}
\frac{d \vec{x}_{p}}{d t} & =\vec{v}_{p} \\
\frac{d \vec{v}_{p}}{d t} & =\frac{q_{p}}{m_{p}}\left(\vec{E}+\vec{v}_{p} \times \vec{B}\right)
\end{aligned}
$$

where $\vec{x}_{p}, \vec{v}_{p}, q_{p}$, and $m_{p}$ are the position, velocity, charge, and mass of the ion particles, and $\vec{E}$ and $\vec{B}$ are the electric and magnetic fields, respectively. The moments of the ions, including the charge density $\left(\rho_{c}\right)$ and the bulk velocity $\left(\vec{u}_{i}\right)$, are gathered by the "Particle in Cell" (PIC) scheme. Meanwhile, the assumptions of mass-less and quasi-neutralization of the electrons are taken into account to calculate the electromagnetic fields as follows:

$$
\begin{aligned}
& \vec{E}=-\vec{u}_{i} \times \vec{B}+\frac{(\nabla \times \vec{B}) \times \vec{B}}{\mu_{0} \rho_{c}}-\frac{\nabla P_{e}}{\rho_{c}} \\
& \frac{\partial \vec{B}}{\partial t}=\nabla \times\left(\vec{u}_{i} \times \vec{B}\right)-\nabla \times\left[\frac{(\nabla \times \vec{B}) \times \vec{B}}{\mu_{0} \rho_{c}}\right]-R \nabla \times\left(\frac{\nabla \times \vec{B}}{\mu_{0}}\right)
\end{aligned}
$$

where $\mu_{0}$ is the permeability of the free space, $P_{e}$ is the electron plasma pressure, and $R$ is the resistivity, which is 0 except inside the Moon. We assume that the plasma pressure would obey the adiabatic law

$P_{e}=P_{e 0}\left(\frac{n_{e}}{n_{e 0}}\right)^{\gamma} \propto \beta_{e 0} \rho_{c}^{\gamma}$

In the simulation, the adiabatic exponent is taken to be $\gamma=2$ (Bagdonat and Motschmann, 2002; Bagdonat, 2004) and the electron plasma-beta $\beta_{e 0}=0.5$, where $\beta_{e 0}$ represents the ratio of the electron plasma pressure to the magnetic pressure $\left(P_{0}=B_{I M F}^{2} /\left(2 \mu_{0}\right)\right.$, and $B_{I M F}$ is the IMF strength). This implies that the electron temperature of the solar wind $\left(T_{e 0}\right)$ is about $7 \times 10^{4} \mathrm{~K}$ in the simulation.

Fig. 1 shows the geometry of the simulations for different orientations of IMF. Three different directions of IMF are chosen with $\theta_{B}=0^{\circ}, 45^{\circ}$, and $80^{\circ}$, where $\theta_{B}$ is the angle of the IMF with respect to $-z$-axis in the $x z$-plane. The dimensions of the simulation box are $240 \times 120 \times 120 c / \omega_{p, i 0}$ where $c$ is the speed of light, $\omega_{p, i 0}$ is the proton plasma frequency in the ambient solar wind, and $c / \omega_{p, i 0}$ is the inertial length of the solar wind proton. In the computation, the simulation box is divided into $240 \times 120 \times 120$ mesh nodes which means that the grid size is $\Delta^{3}=1 \times 1 \times 1 \mathrm{c} / \omega_{p, i 0}$. The time step is $\Delta t=0.01 \Omega_{i 0}^{-1}$, where $\Omega_{i 0}$ is the gyrofrequency of the solar wind protons. The upstream solar wind parameters are given as $\left|\vec{B}_{I M F}\right|=5 \mathrm{nT}$, the proton number density $\left(n_{s w 0}\right)=5 \mathrm{~cm}^{-3}$, and the solar wind speed $\left(V_{s w 0}\right)=400 \mathrm{~km} / \mathrm{s}$. This would lead the Debye length of the solar wind in the simulation to be $\approx 8 \mathrm{~m}$ $\left(\lambda_{s w 0}=\sqrt{\varepsilon_{0} k T_{e 0} / n_{s w 0} e^{2}}\right.$, where $\varepsilon_{0}$ is the permittivity of the free space, $k$ is the Boltzmann constant, and $e$ is the electronic charge), the gyrofrequency of the solar wind proton $\left(\Omega_{i 0}\right) \approx 0.48 \mathrm{~s}^{-1}$, the Alfvén speed of the solar wind $\left(V_{A 0}=\left|\vec{B}_{I M F}\right| / \sqrt{\mu_{0} m_{p} n_{s w 0}}\right) \approx$ $48.8 \mathrm{~km} \mathrm{~s}^{-1}$, and the inertia length of the solar wind proton $\left(c / \omega_{p, i 0}\right) \approx 102 \mathrm{~km}$.

To initialize the calculation, the magnetic fields at all the mash nodes are set as the IMF including inside the Moon, and each cell in the simulation box except inside the obstacle is filled with 20 macro-particles. All the particles are randomly generated 


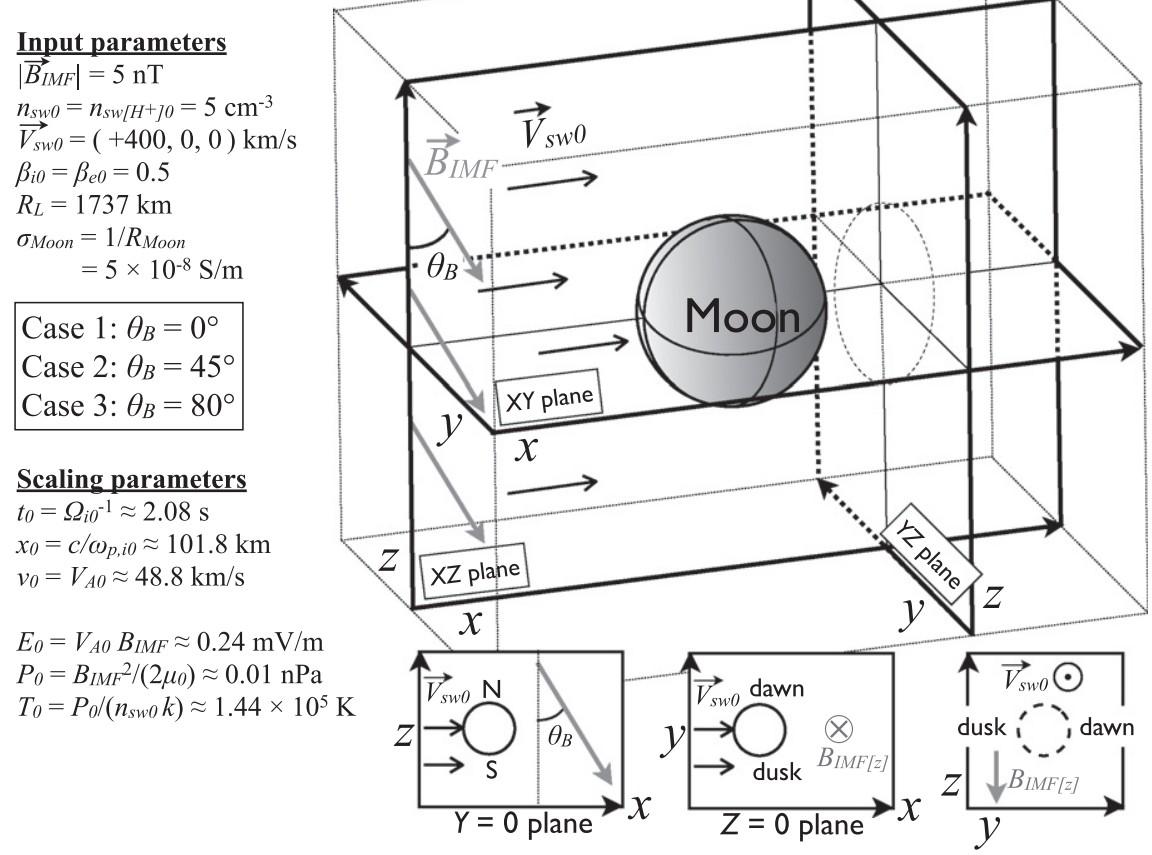

Fig. 1. The initialization of the simulation box and the input parameters. The three boxes at the bottom demonstrate the geometry of the displayed results.

according to the Maxwellien velocity distribution with the ion plasma-beta value $\beta_{i 0}=0.5$, which is assumed to be equal to the electron plasma-beta. Therefore, in addition to the bulk solar wind velocity, the thermal speed of the ions $\left(V_{t h}\right)$ is about $42.3 \mathrm{~km} / \mathrm{s}$. If a particle hits the lunar surface, it will be removed from the calculation. For each time step, all the particles inside the cells of the upwind boundary in the $x$-direction is replaced with undisturbed solar wind ions, and the magnetic fields are fixed as the IMF. For the downwind boundary in the $x$-direction, the particles are copied from the inner cells, and the magnetic fields are extrapolated from the inner grid nodes with a zeroth order approximation. Both sides of the $y$ and $z$ boundaries are set to be periodic. We also test with other kinds of boundaries, which turn out not to have important effects on the wake structures.

\section{The lunar conductivity}

To examine the lunar influence on the wake structure, the magnetic fields inside the Moon can be calculated with the last term in Eq. (4) by changing the resistivity $(R)$. In the numerical simulations, the boundary at the lunar surface divided the regions of zero resistivity in the solar wind and finite resistivity $(R)$ in the interior is treated by using a profile as illustrated in Fig. 2. A smoothed curve is needed to ensure that computational stability is afforded by the grid sizes used. Some numerical effect will occur near to the lunar surface due to the smoothing treatment of the obstacle.

On the sunward side, each solar wind macro-particle impinging on the lunar surface will be removed. As a result, no particles will exist in the near wake region in the simulation. Since we use magneto-hydrodynamic (MHD) treatment for the electromagnetic fields in the hybrid model (see Eqs. (3) and (4)), the magnetic field lines should be bounded to the plasma, with no significant dissipation occurring in the free space in the near wake region. With the lack of plasma, the convection of the magnetic field lines will be suspended. Therefore, the field lines will pile up on the lunar night-side in the model. This discrepancy between the hybrid

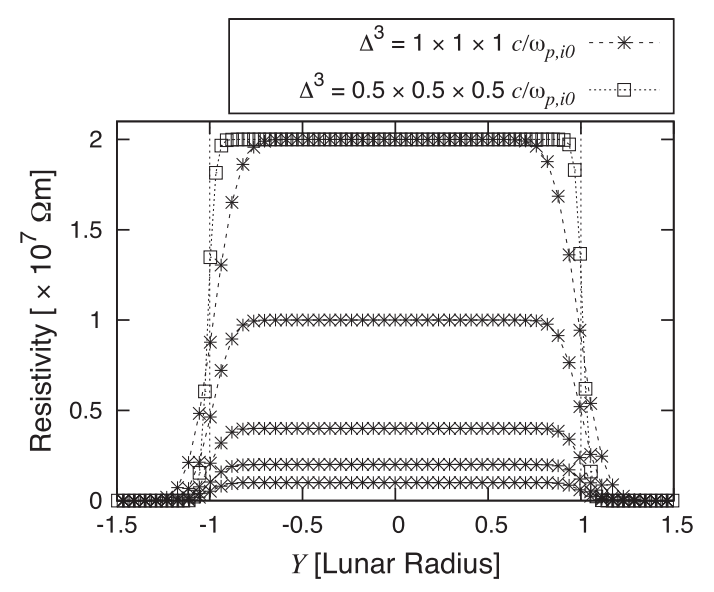

Fig. 2. The resistivity $(R)$ distributions of the Moon used in different simulation cases. The profiles with asterisks are for cases with the grid size $\Delta^{3}=1 \times 1 \times 1$ $c / \omega_{p, i 0}$, and that with squares is for $\Delta^{3}=0.5 \times 0.5 \times 0.5 c / \omega_{p, i 0}$.

simulation and the measurements arises from the fact that the extremely low number density would lead to a very large Debye length $\left(\lambda_{0} \propto n_{i}^{-1 / 2}\right)$ in the near wake region. The MHD description of the electromagnetic fields will fail with the present grid size system used in the model. To achieve the MHD assumption on the anti-sunward side, a stream of macro-particles is emitted along the $x$-direction from the night-side. As suggested by the measurements (Schubert and Lichtenstein, 1974; Colburn et al., 1967; Owen et al., 1996), the solar wind speed is chosen for the wake ions. The relative number density of these macro-particles is taken to be only $\sim 10^{-4}$ of the upstream value, leading to a Debye length of $\sim 800 \mathrm{~m}$ in the wake region. The MHD consideration will now work better. The difference between the number densities inside and outside the wake is large enough (about 4 orders of magnitude) that the influence of the wake protons so injected is relatively small. 

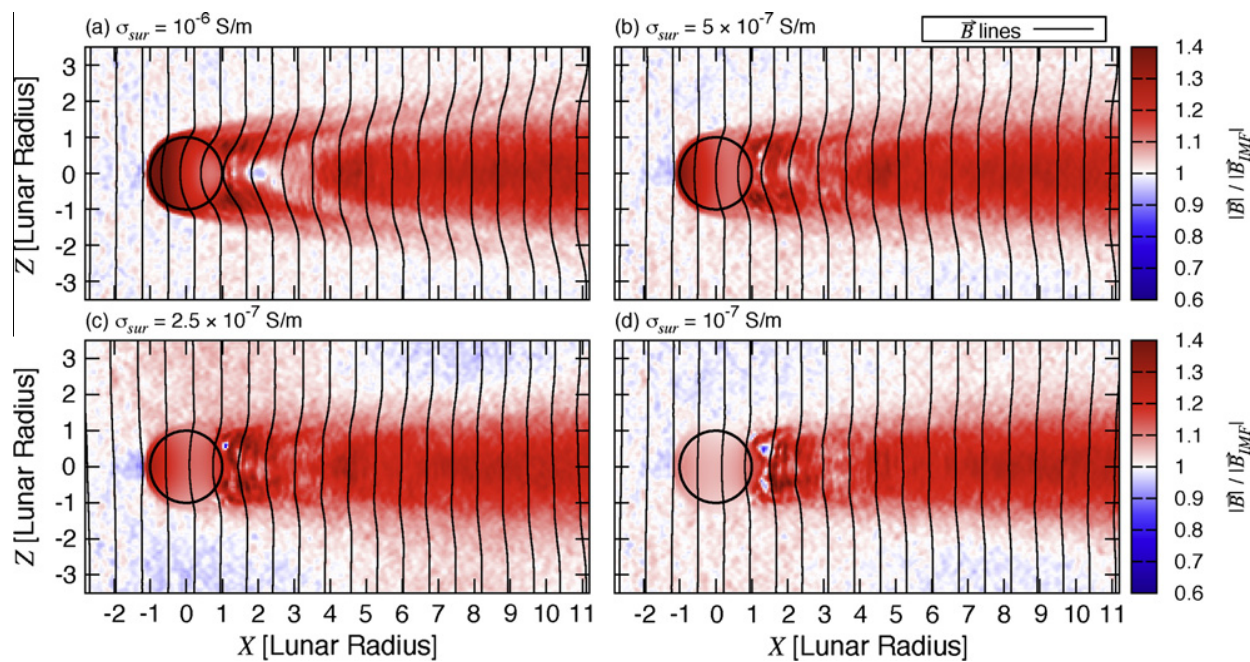

Fig. 3. The magnetic field strength $(|\vec{B}|)$ distributions and the magnetic field lines in the $x z$-plane for different conductivity $(\sigma=1 / R)$ cases for the Moon when $\theta_{B}=0^{\circ}$.

In the next step, different lunar resistivities are chosen to test their influence on the wake region when $\theta_{B}$ is set to $0^{\circ}$. Fig. 3 shows the results for the magnetic field strength and field line distributions in the $x z$-plane. If the conductivity $(\sigma=1 / R)$ is large enough, currents $(\nabla \times \vec{B})$ could be generated inside the Moon, leading to the deformation of the field lines (Fig. 3a-c). Similar effects could be observed for Venus and Mars with a conducting ionosphere (Bößwetter et al., 2004). When the conductivity is as small as $10^{-7} \mathrm{~S} / \mathrm{m}$ (Fig. 3d), the magnetic field draping vanishes and the free passage of the field lines will be accomplished. We can conclude that a conductivity as low as $10^{-7} \mathrm{~S} / \mathrm{m}$ is small enough to stop significant current generation (Glassmeier, 2000; Janhunen and Kallio, 2004). In this situation, both the numerical electric field $(\vec{E})$ and the time variation of the magnetic field $(\partial \vec{B} / \partial t)$ inside the Moon will be nearly 0 (see Eqs. (3) and (4)). Thus, we use a lunar conductivity of $5 \times 10^{-8} \mathrm{~S} / \mathrm{m}$ in the following simulations.

\section{Magnetic fields in the lunar wake}

In our hybrid simulations, a quasi-steady condition is reached after 3000 time steps, when the solar wind has moved past the length of the simulation box about one time. We will first discuss the wake structure when $\theta_{B}=0^{\circ}$. Fig. $4 \mathrm{a}$ and $\mathrm{b}$ shows the distributions of the normalized values of the solar wind proton density around the Moon in the $x z$ - and $x y$-planes, respectively. It can be seen in Fig. 4a that because of the assumption of total absorption of the solar wind at surface impact, a near-vacuum is formed in the wake region. The magnetic field lines move unimpeded through the lunar interior under the assumption that the Moon is a poor conductor. The protons in the solar wind move along the field lines with a thermal speed of about $42.3 \mathrm{~km} / \mathrm{s}\left(V_{t h}\right)$, therefore there is a gradual refilling of the lunar wake leading to the formation of a conical structure in the low density core (i.e., the deep blue region) surrounded by a sheath of intermediate density (i.e., the green zone). The absorption effect extends a large downstream distance beyond $11 R_{L}$. As for the distributions of the plasma parameters in the $x y$-plane, we find a similar low density region in the center of the wake in Fig. $4 \mathrm{~b}$ when compared to the $x z$-plane distribution in Fig. 4a. A pair of rarefaction zones also forms which envelope the lunar wake. The asymmetric structure is the result of the influences of the different directions in which the electric fields point. The formation of the electric fields would be discussed in Section 5 .
Fig. $4 \mathrm{c}$ and $\mathrm{d}$ shows variations of the magnetic field strength in the $x z$ - and the $x y$-planes, respectively. In Fig. 4c, we find enhancement of the magnetic field strength in the lunar wake in response to the depletion of the plasma density. To reach the pressure equilibrium, the maximum strength is about 1.4 times the value of the upstream solar wind. This enhancement is comparable to the detected values (Colburn et al., 1967; Ness et al., 1968; Schubert and Lichtenstein, 1974; Halekas et al., 2005). In Fig. 4d which is perpendicular to the IMF direction, we find that the central lunar wake has an area of enhanced magnetic field strength sandwiched between a pair of field-reduction zones. This can be understood in terms of the bending of the magnetic field lines towards the wake center (Michel, 1968; Owen et al., 1996). Fig. 4e and f shows the speed and the streamline distributions of the solar wind and the wake protons. Larger inward diffusion can be found along the magnetic field lines in the $x z$-plane than perpendicular to the field lines in the $x y$-plane. The anisotropic expansion of the solar wind plasma into the wake region, due to the orientation of the magnetic field lines, can be easily observed.

Fig. 5 shows the patterns of the magnetic field strength and the proton number density distributions at different distances from the Moon (i.e., $X=2$ and $6.5 R_{L}$ ). The inward bending of the field lines occurs in response to the reduction in the plasma pressure. The refilling of the solar wind plasma in the wake (see Fig. 5c and d) is partly facilitated by this motion of the field lines. The diamagnetic currents can be generated in the rarefaction region of the magnetic field strength through the plasma pressure gradient $\left(\vec{j}_{\text {dia }}=\vec{B} \times \nabla P / B^{2}\right)$. The enhancement of the magnetic field strength in the center could be considered to arise as a result of Ampère's law (Owen et al., 1996).

In the simulation, when $\theta_{B}=0^{\circ}$, the rarefaction wave of the magnetic fields induced by the inward filling of the wake could extend outward and form a Mach cone with an open angle of around $10^{\circ}$. For comparison, Fig. 6 shows the magnetic field distributions in the $x z$ - and $x y$-planes for $\theta_{B}=45^{\circ}$ and $80^{\circ}$. Note that the Mach angles are about the same value given different IMF orientations. We can conclude from this that the Mach cone may not depend on the IMF orientation. This angle is a little larger than the average value from the measurements, which is about $8^{\circ}$ (Whang and Ness, 1970). The rarefaction wave propagates as either a sonic wave or fast-mode magnetosonic wave. When the sonic speed of the solar wind is $48.8 \mathrm{~km} / \mathrm{s}\left(V_{S 0}^{2}=\gamma\left(\beta_{i 0}+\beta_{e 0}\right) V_{A 0}^{2} / 2=V_{A 0}^{2}\right.$, with $\gamma=2$ and $\beta_{i 0}=\beta_{e 0}=0.5$ in the simulation) and the fast-mode magnetosonic speed is $69 \mathrm{~km} / \mathrm{s}\left(V_{F 0}^{2}=V_{S 0}^{2}+V_{A 0}^{2}\right)$, the Mach cone angles with 
(a) $n_{H^{+}} / n_{s w 0}$

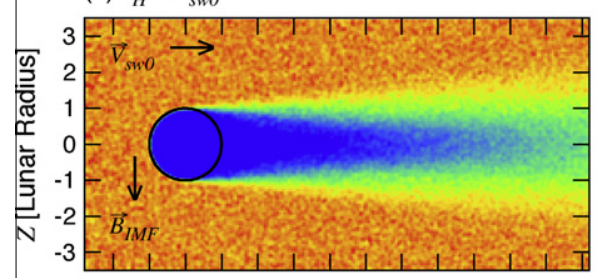

(c) $|\vec{B}| /\left|\vec{B}_{I M F}\right|$

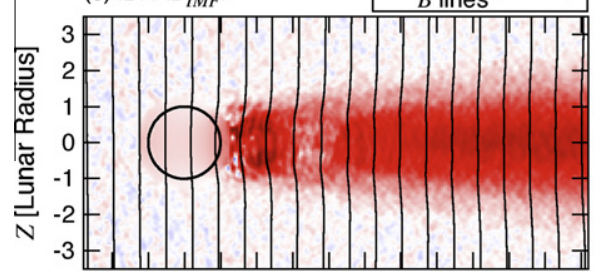

(e) $|\vec{U}|[\mathrm{km} / \mathrm{s}]$

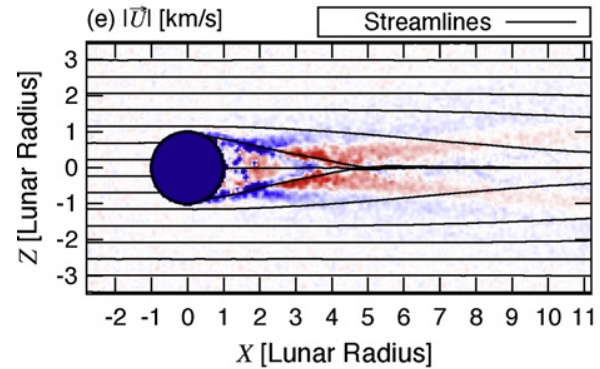

(b) $n_{H^{+}} / n_{s w 0}$

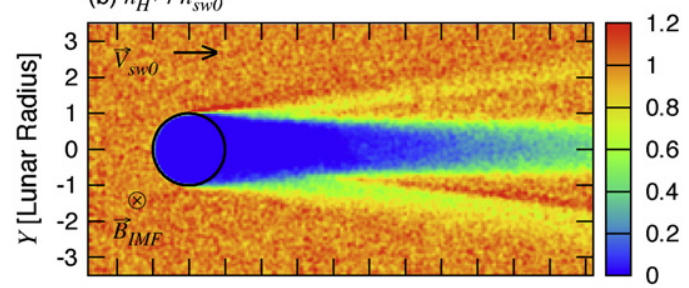

(d) $|\vec{B}| /\left|\vec{B}_{I M F}\right|$
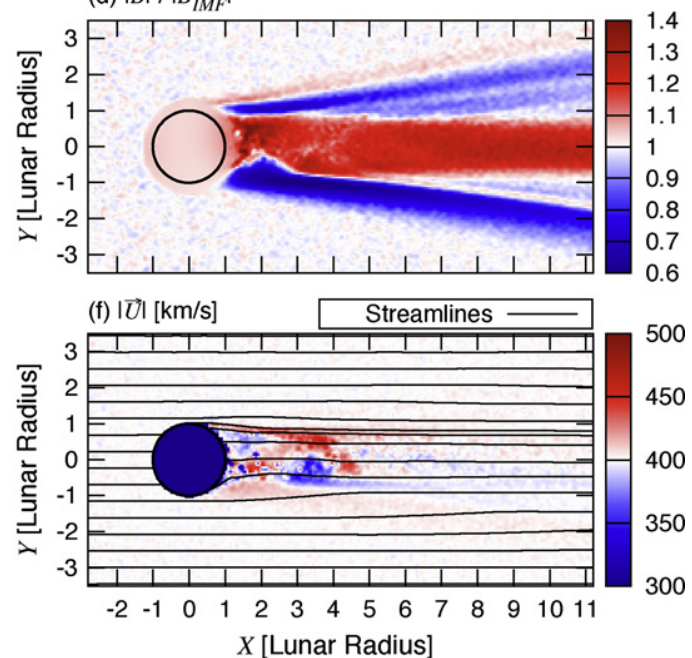

Fig. 4. The solar wind proton number density $\left(n_{H^{+}}\right)$, the magnetic field strength $(|\vec{B}|)$, and the bulk speed $(|\vec{U}|)$ distributions in the $x z-$ and $x y$-planes when $\theta_{B}=0^{\circ}$.

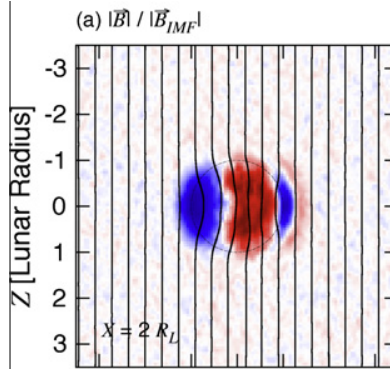

(b) $|\vec{B}| /\left|\vec{B}_{I M F}\right|$

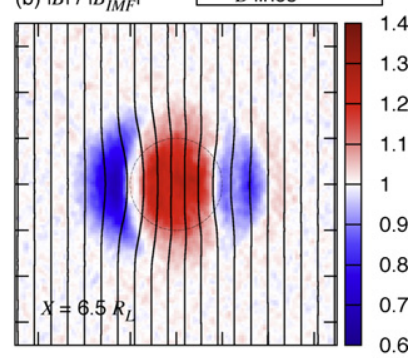

(c) $n_{H^{+}} / n_{s w 0}$

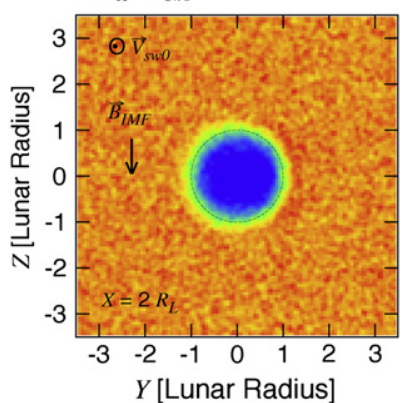

(d) $n_{H^{+}} / n_{\text {swo }}$

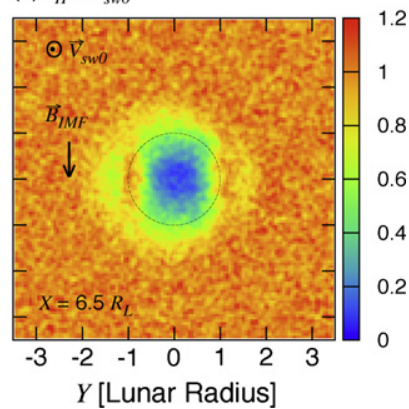

Fig. 5. The magnetic field strength $(|\vec{B}|)$, magnetic field lines, and solar wind proton number density $\left(n_{\mathrm{H}^{+}}\right)$distributions in different segments of the $y z$-planes when $\theta_{B}=0^{\circ}$.

respect to the solar wind speed are about $7^{\circ}$ and $10^{\circ}$, respectively. This suggests that the formation of the Mach cone depends on the magnetosonic speed in our hybrid model. This is consistent with the simulation results of Holmström et al. (2011).

Fig. 7 shows the magnetic field strength distribution along the $z$ - and $y$-directions at different lunar distances $\left(X=2\right.$ and $\left.6.5 R_{L}\right)$ for $\theta_{B}=0^{\circ}, 45^{\circ}$, and $80^{\circ}$. The "upstream" labels indicate the connec- tion with the upstream solar wind through the magnetic field lines and the "downstream" ones are the downstream related when $\theta_{B}>0^{\circ}$ (see Fig. 6a and c). The overall magnetic field strength distribution essentially follows the observational results of spacecraft measurements (Ness et al., 1968; Schubert and Lichtenstein, 1974; Halekas et al., 2005), which includes an enhancement in the center of the wake of about $30-40 \%$ of the IMF value and two depletion dips on the two sides of about $-20 \%$ of the solar wind. No obvious 'limb shock' enhancements outside the dips can be discerned in the simulation. The origin of the limb shock has been suggested to be the result of the interaction of the solar wind with the crustal remnant magnetic fields (see Schubert and Lichtenstein (1974) and the references therein). This interaction lies outside the scope of this paper. Simulations with the inclusion of the magnetic remnants will be needed in future for closer examination of the limb shock scenario.

For different IMF orientations, the magnetic field enhancements in the center of the wake are similar to one another at $X=2 R_{L}$. However, the enhancement decreases toward the tail (at $X=6.5 R_{L}$ ) when $\theta_{B}=0^{\circ}$, but increases when $\theta_{B}=80^{\circ}$. This is because the solar wind plasma can diffuse inward faster when the magnetic field lines are perpendicular to the wake, i.e., $\theta_{B}=0^{\circ}$. As a consequence, the number density in the wake also increases faster. In order to maintain the pressure balance, the magnetic field strength will respond to the density variation.

The motion of the solar wind protons in the wake is controlled by the field-aligned thermal diffusion process as well as acceleration by the electric fields established at the wake boundaries. The combination of both effects leads to asymmetry in the plasma distribution which will be analyzed in Section 5 .

\section{Electric fields in the lunar wake}

As mentioned before, because of the surface absorption of the solar wind plasma, a near-vacuum is created immediately behind 

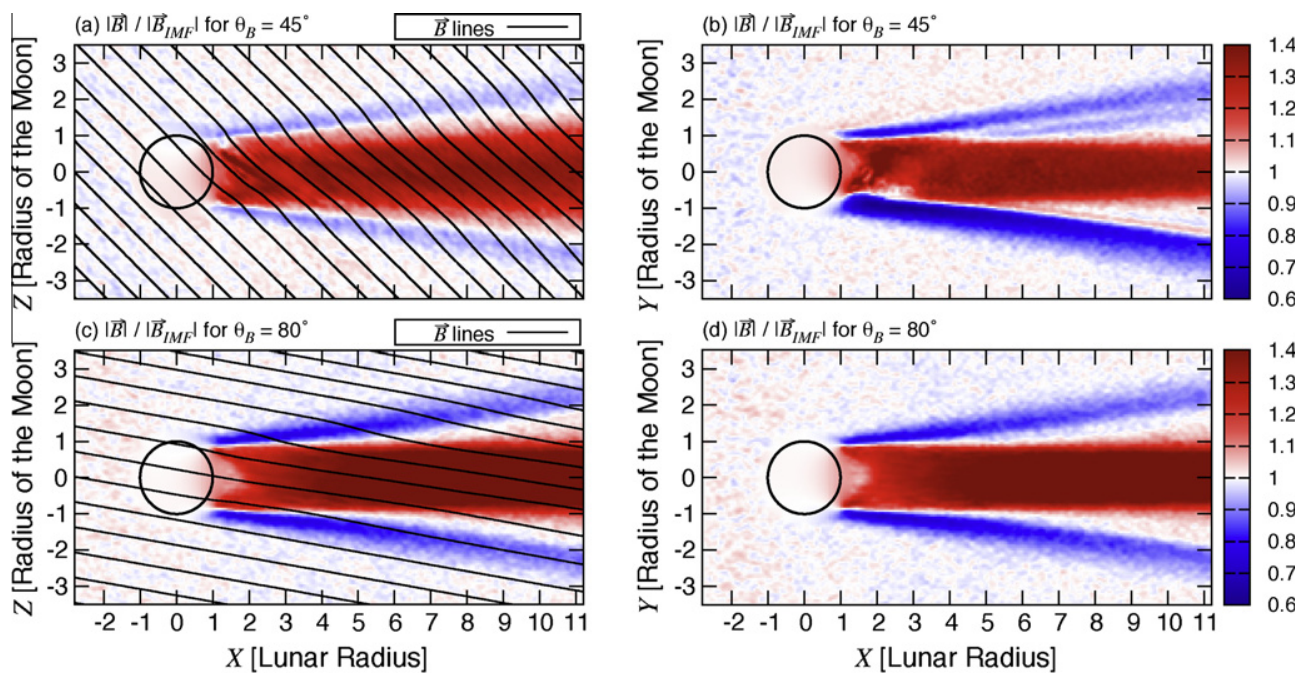

Fig. 6. A comparison of the magnetic field strength $(|\vec{B}|)$ distribution in the $x z$ - and $x y$-planes for $\theta_{B}=45^{\circ}$ and $80^{\circ}$.
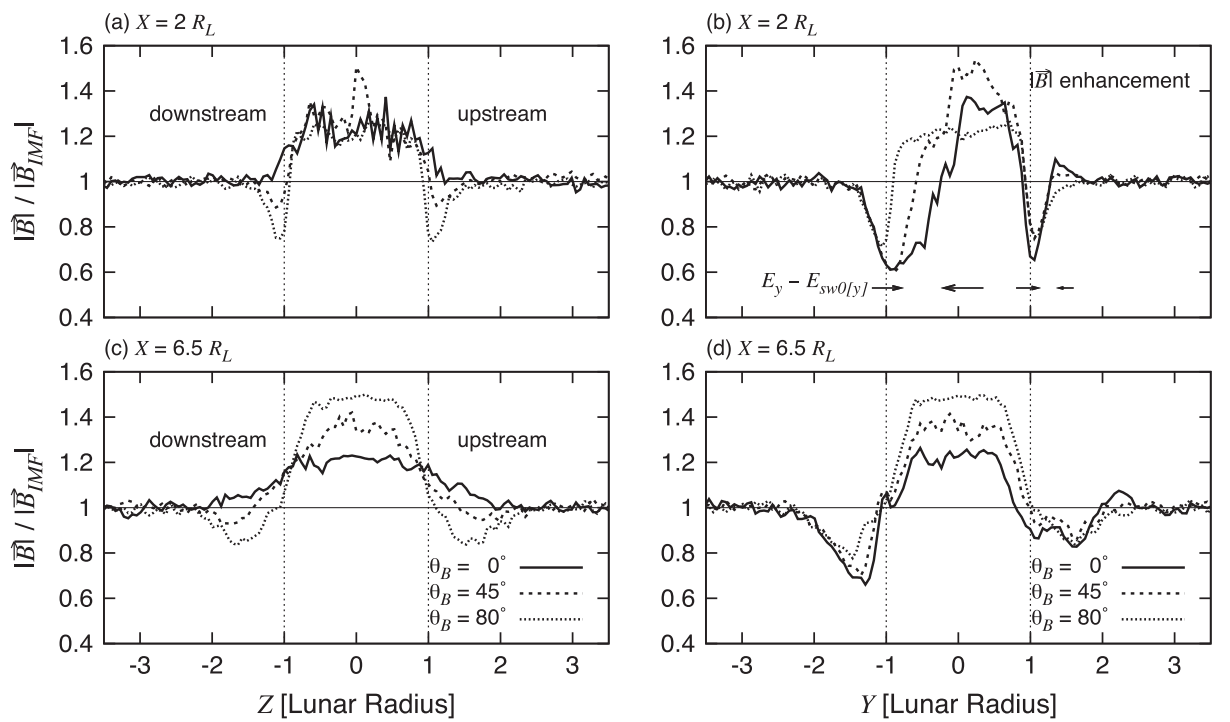

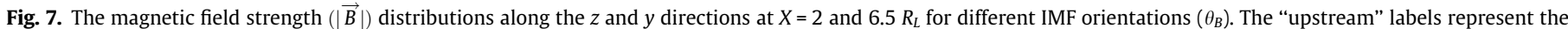
wake connection with the upstream solar wind through the magnetic field lines and the "downstream" ones relate to the downstream parts when $\theta_{B}>0^{\circ}$.

the night-side hemisphere. The kinetics of the fast-streaming of the electrons along the magnetic field lines will lead to the establishment of an electric field at the boundary of the lunar wake (Birch and Chapman, 2001, 2002). The beams of counter-streaming solar wind protons were observed by the plasma experiment on the Wind spacecraft as it moved across the lunar wake at a distance of $6.5 R_{L}$ from the Moon (Ogilvie et al., 1996). The strength of the electrical field was estimated to be about $0.2 \mathrm{mV} / \mathrm{m}$. In the present treatment of the electrons as a neutralizing fluid, no charge separation effect exists in the hybrid model calculations. Instead, the electric field can be approximated by using Eq. (3) which contains the convective electric field $\left(-\vec{u}_{i} \times \vec{B}\right)$ associated with the bulk flow of the solar wind, the $\nabla \times \vec{B}$ term and the electron plasma pressure gradient $\left(\nabla P_{e}\right)$ term. This means that in the upstream region, the electric field will be given by $\left|\vec{E}_{s w 0}\right|=\left|-\vec{u}_{s w 0} \times \vec{B}\right|$ $\left(\approx 2 \mathrm{mV} / \mathrm{m}\right.$ for $\left.\theta_{B}=0^{\circ}\right)$ pointing in the $-y$-direction, because both the $\nabla \times \vec{B}$ and $\nabla P_{e}$ terms are zero. However, in the vicinity of the lunar wake all these terms will contribute to the electric fields.

Fig. 8 shows the distributions of the electric fields for components in the $z$ - and $y$-directions, respectively, given different IMF orientations. Fig. 8a shows the electric field distributions in the $x z$-plane with $\theta_{B}=0^{\circ}$. The $z$-component of the electric fields at the wake boundaries, which are pointing towards the wake center is about $0.5 \mathrm{mV} / \mathrm{m}$ in magnitude. This theoretical value is comparable to the value estimated by Ogilvie et al. (1996) from their Wind measurements. However, the directions of the fields change about two times inside the wake. The situation becomes even more complicated when $\theta_{B}=45^{\circ}$ (Fig. 8c). While the "upstream" parts exhibit similar pointing directions as when $\theta_{B}=0^{\circ}$, there is an outward oriented electric field that envelopes around the outer edge of the wake at larger distances down tail on the "downstream" side. Gradually, the outward pointing direction of the electric fields appears more obvious at the wake boundary at the "downstream" side as $\theta_{B}=80^{\circ}$ (Fig. 8e).

The resulting electric field distribution is generated by a combination of the $\left(-\vec{u}_{i} \times \vec{B}\right), \nabla \times \vec{B}$ and $\nabla P_{e}$ terms. Of these, the first term for the nature of the convective electric field contributes the most in our analysis. Since the bulk velocities of the ions are dominated by the original solar wind speed in the $+x$-direction (see Fig. $4 \mathrm{e}$ and $\mathrm{f}$ ), the formation of the $z$-component of the electric 
(a) $\theta_{B}=0^{\circ}$

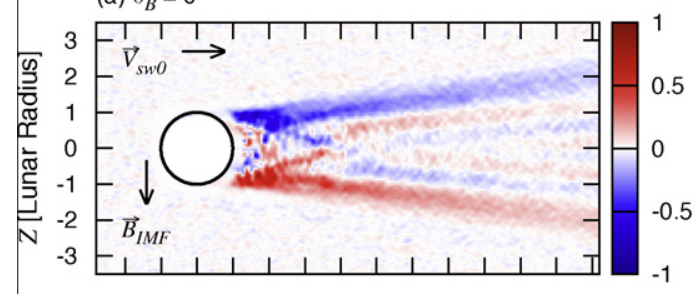

(c) $\theta_{B}=45^{\circ}$

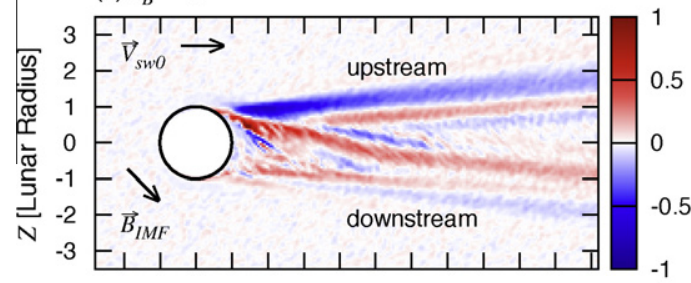

(e) $\theta_{B}=80^{\circ}$

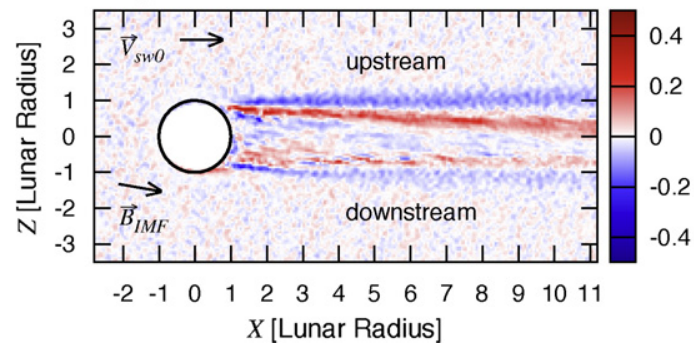

(b) $\theta_{B}=0^{\circ}$

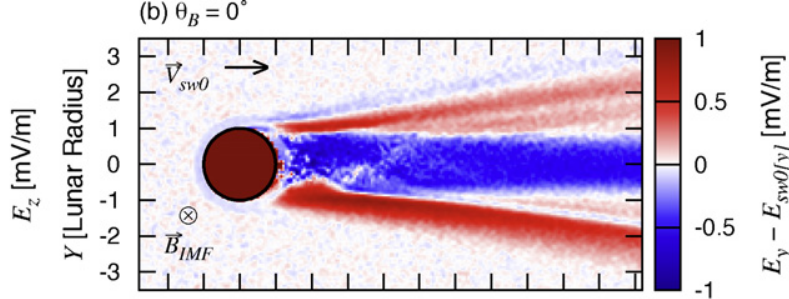

(d) $\theta_{B}=45^{\circ}$

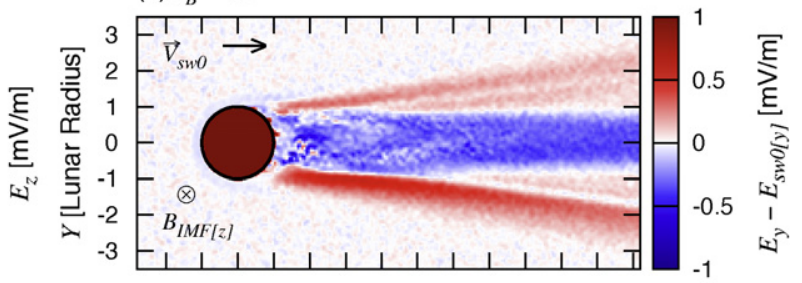

(f) $\theta_{B}=80^{\circ}$

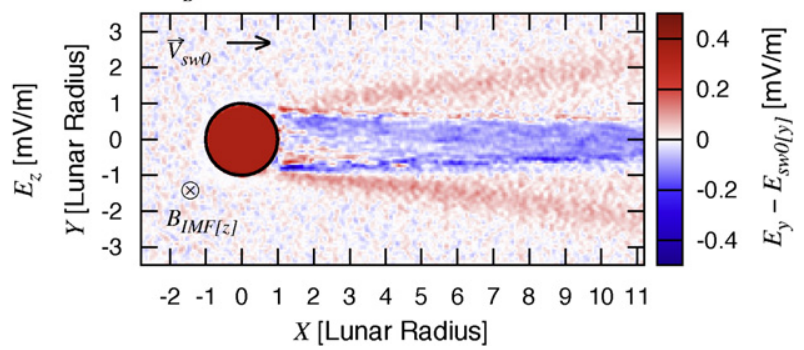

Fig. 8. The $z$ - and $y$-components of the electric field $(\vec{E})$ distributions in the $x z$ - and $x y$-planes, respectively, for different IMF orientations $\left(\theta_{B}\right)$.

field comes from the bending of the $-z$-pointing magnetic field lines in the $y$-direction. On the other hand, the generation of the $y$-component of the electric fields will mostly result from the enhancement or depletion of the $-z$-pointing magnetic field strength directly. Fig. 8b, d, and f show the $y$-component of the electric field in the $x y$-plane obtained by subtracting the theoretical value of the convective solar wind electric field $\left(\vec{E}_{s w 0}\right)$ as given by Eq. (3). Compared with Figs. $4 \mathrm{~d}$ and $6 \mathrm{~b}$, d, it can be seen that the structures of the $y$-component of the electric field are similar to the magnetic field strength distributions.

Fig. 9 shows the transition of the distributions of the strength of the magnetic field and the induced electric field at a distance of $6.5 R_{L}$ from the lunar center in the $y z$-plane for $\theta_{B}=0^{\circ}$ to $80^{\circ}$. As would be expected, the pattern of magnetic field enhancement shifts from a sandwich-type configuration (Fig. 9a) to a banquette-type configuration (Fig. 9c). A comparison of the changes of the electric field strength (pointing away from the wake center) can be found in Fig. 9d-f. As $\left(\vec{u}_{i} \times \vec{B}\right)$ gradually diminishes from $\theta_{B}=0^{\circ}$ to $80^{\circ}$, the induced electric field also decreases in magnitude. Although we might expect that the patterns of depletion in magnetic field strength on both sides of the wake would be symmetrical along the $y$-direction, the resultant electric fields will point in different directions with respect to the wake center. Consequently, asymmetries will occur in the diffusion of the plasma, in relation to the magnetic fields. We can see obvious dissymmetries in the magnetic field strength distribution in Fig. $7 \mathrm{~b}$ and $\mathrm{d}$. On the other hand, asymmetrical features also take place along the field lines when $\theta_{B}>0^{\circ}$. We can expect the plasma motions to have different behaviors on the "upstream" and "downstream" sides.

Fig. 10 shows the distributions of the magnetic field strengths, the proton number densities, the $z$-component of the electric fields, the bulk speeds and the $z$-component of the bulk velocities of the protons along the $z$-direction for different IMF orientations at $X=6.5 R_{L}$. When $\theta_{B}=0^{\circ}$, no obvious asymmetry can be observed in the velocities of the protons. The ions could diffuse inward from both sides of the wake to be accelerated by the inward pointing electric fields (see $U_{H^{+}[z]}$ and $\left|\vec{U}_{H^{+}}\right|$in Fig. 10a.4). As the tilt of the angle in the IMF directions departs increasingly from perpendicular to the solar wind flow, differences in diffusion along both ends of the magnetic field lines will appear. The "upstream" diffusions will be accelerated by inwardly oriented electrical fields at the wake boundary, which is similar to the case with $\theta_{B}=0^{\circ}$ but speeded up. On the contrary, inward diffusion from the "downstream" side along the magnetic field lines is more difficult. A certain level of acceleration at the wake boundary could be generated. However, the protons must decelerate against the "downstream"ward bulk flow in order to diffuse "upstream" toward the center of the wake along the field lines. The case with $\theta_{B}=45^{\circ}$ exhibits velocity and speed features similar to the Wind observations. The inbound trajectory indicates the "upstream" side of the wake and the outbound refers to the "downstream" one (Fig. 1 in Ogilvie et al. (1996)). Although the hybrid code does not include electric fields induced by charge separations, the convective electric fields could serve nearly the same function.

\section{Discussion}

Recently, there are prominent satellite missions to the Moon, and most of them aim to the near lunar surface environments with relatively low altitude detections. One is satellite Kaguya with polar orbits of $\sim 100 \mathrm{~km}$ from the lunar surface. From the latest Kaguya measurements, the solar wind entry to the night-side could occur down to $46-33^{\circ}$ in latitude (Nishino et al., 2009b). Under specific solar wind conditions, including slow solar wind and dawn-dusk pointing IMF, energy gain and loss can be detected from the solar protons behind the terminators. This is called the 'Type-I entry' of the solar wind protons into the near lunar wake region (Nishino et al., 2009a,b). In order to study such a deep entry effect at low altitude, another simulation is performed using $(1 / 2)^{3}$ 
(a) $\theta_{B}=0^{\circ}$

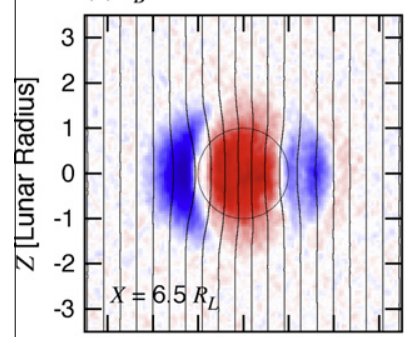

(d) $\theta_{B}=0^{\circ}$

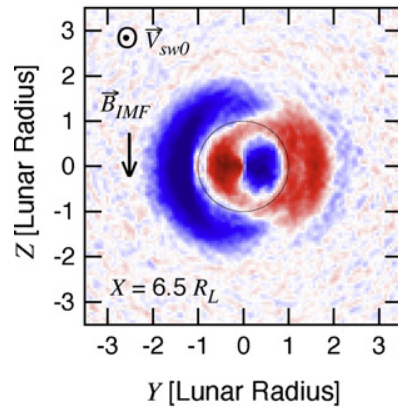

(b) $\theta_{B}=45^{\circ}$

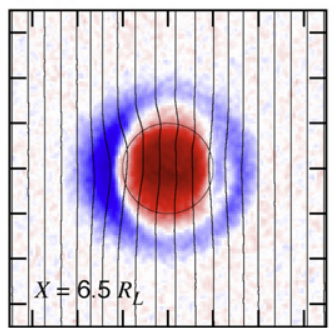

(e) $\theta_{B}=45^{\circ}$

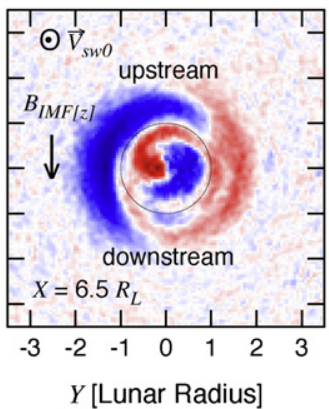

$\vec{B}$ lines through $Z=0$

(c) $\theta_{B}=80^{\circ}$

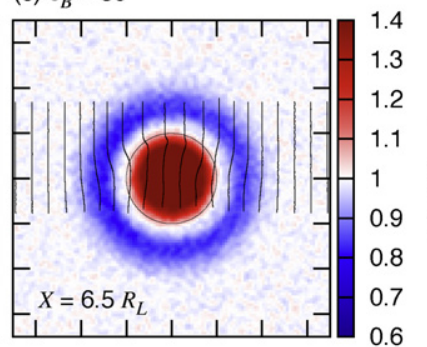

(f) $\theta_{B}=80^{\circ}$

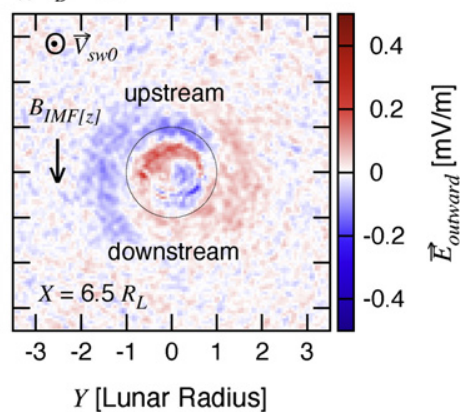

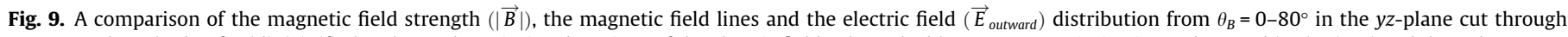

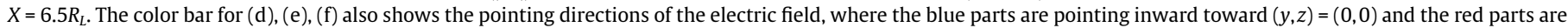
pointing outward away from $(y, z)=(0,0)$. (For interpretation of the references to colour in this figure legend, the reader is referred to the web version of this article.)
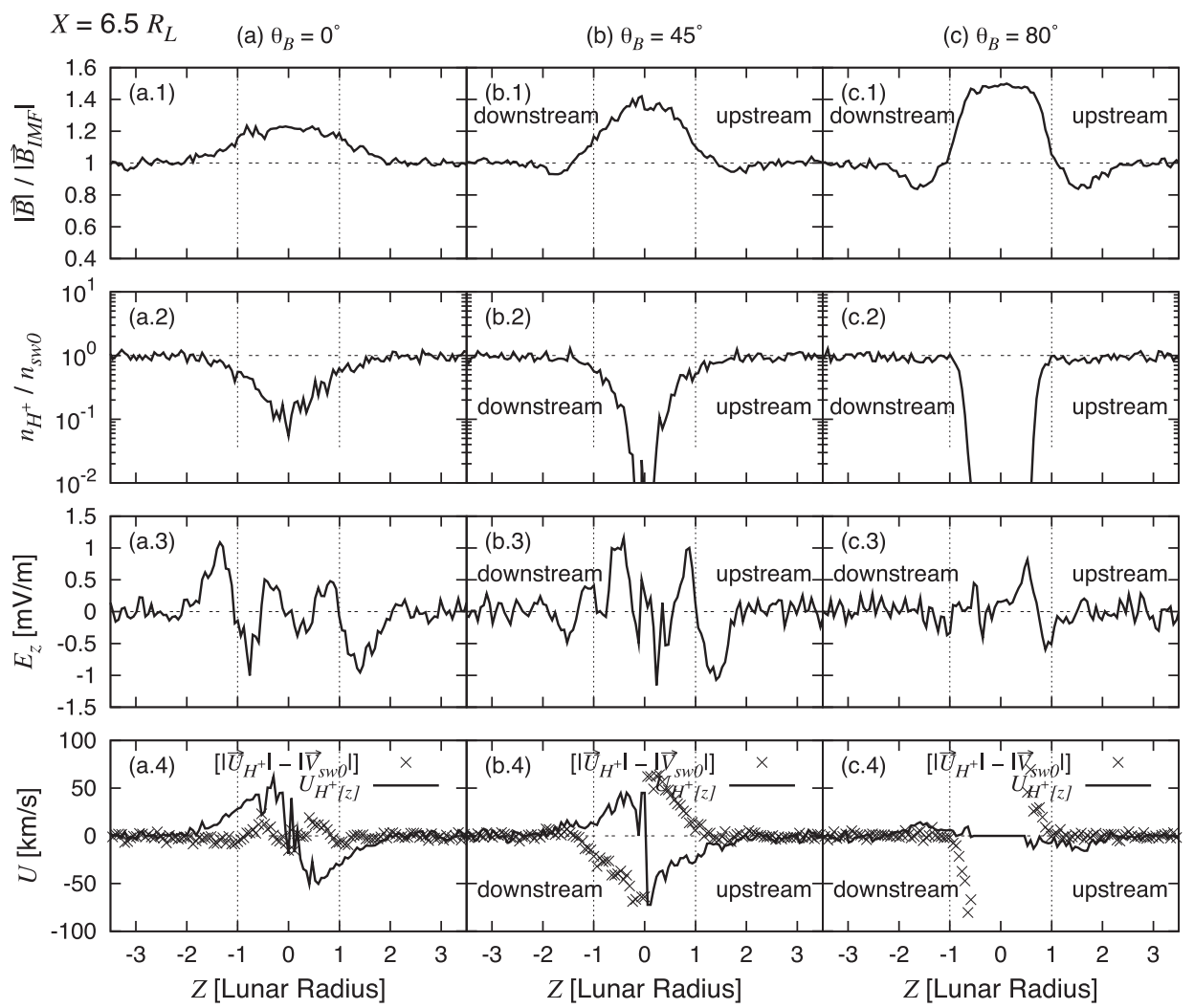

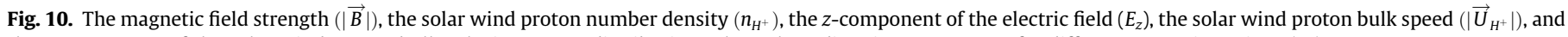
the $z$-component of the solar wind proton bulk velocity $\left(U_{H^{+}[z]}\right)$ distributions along the $z$ direction at $X=6.5 R_{L}$ for different IMF orientations $\left(\theta_{B}\right)$.

the original grid size in cubic form $\left(\Delta^{3}=0.5 \times 0.5 \times 0.5 c / \omega_{p, i 0}\right)$ for $\theta_{B}=0^{\circ}$. The simulation box is smaller in size to $70 \times 70 \times 70 \mathrm{c} / \omega_{p, i 0}$. The time step is also reduced to half the original one $(\Delta t=0.005$ $\Omega_{i 0}^{-1}$ ) and the quasi-steady state condition can be reached after
2500 time steps. The lunar resistivity profile $\left(R=2 \times 10^{7} \Omega \mathrm{m}\right)$ is shown in Fig. 2. Other parameters are set to be the same as the original ones. Fig. 11 shows a comparison of the numerical results at $X=2 R_{L}$ obtained with these two different grid sizes. While we have 
the same number of solar wind macro-particles per cell, the statistics become better as the mesh nodes increase in the same space. As a result, we will achieve lower numerical noise in the simulation as the resolution increases. The fluctuations inside the wake region in the flow patterns (Fig. $4 \mathrm{e}$ and $\mathrm{f}$ ) and the electric fields (Fig. 8a, c, and e) might be the result of the numerical noise, since we have even smaller numbers of macro-particles inside the wake. Nevertheless, the electromagnetic field distributions at the wake boundaries show the same trends for different grid size simulations, as do the flow patterns.

A special solar wind condition is needed for a better comparison with the 'Type-I entry' effect reported in Nishino et al. (2009b). Thus we use a slower solar wind speed of $V_{s w 0(s)}=342 \mathrm{~km} / \mathrm{s}$. The other parameters in the simulation remain the same $\left(\theta_{B}=0^{\circ}\right)$. Fig. 12 shows the $y$-component of the electric field distribution in the $x y$-plane. Because the electromagnetic fields are affected by the smoothing profile of the lunar resistivity up to an altitude of $\sim 250 \mathrm{~km}$ from the surface, we therefore exclude this layer and trace some particular solar wind proton trajectories. Note that the trajectories of different cases are from the results of different simulations. Without switching the IMF geometry, the entry of the solar wind from the south and north pole in the dawn-dusk pointing IMF environment (from the measurements) is compared with dawn-dusk side entry ( $y$-direction) as the IMF points southward ( $z$-direction) in the simulation. Given the same thermal speed ( $V_{t h} \approx 42.3 \mathrm{~km} / \mathrm{s}$ ), deeper entry of the protons into the wake can be found for slower solar wind case $\left(V_{s w 0(s)}\right)$. Inward pointing electric fields could be found with magnitudes of about 0.2$0.3 \mathrm{mV} / \mathrm{m}$ at both sides of the wake boundaries. Outward pointing electric fields, however, exist inside the inward pointing ones at the dawn-side ( $Y \approx 1$ in Fig. 12 ). This is different from the assump-

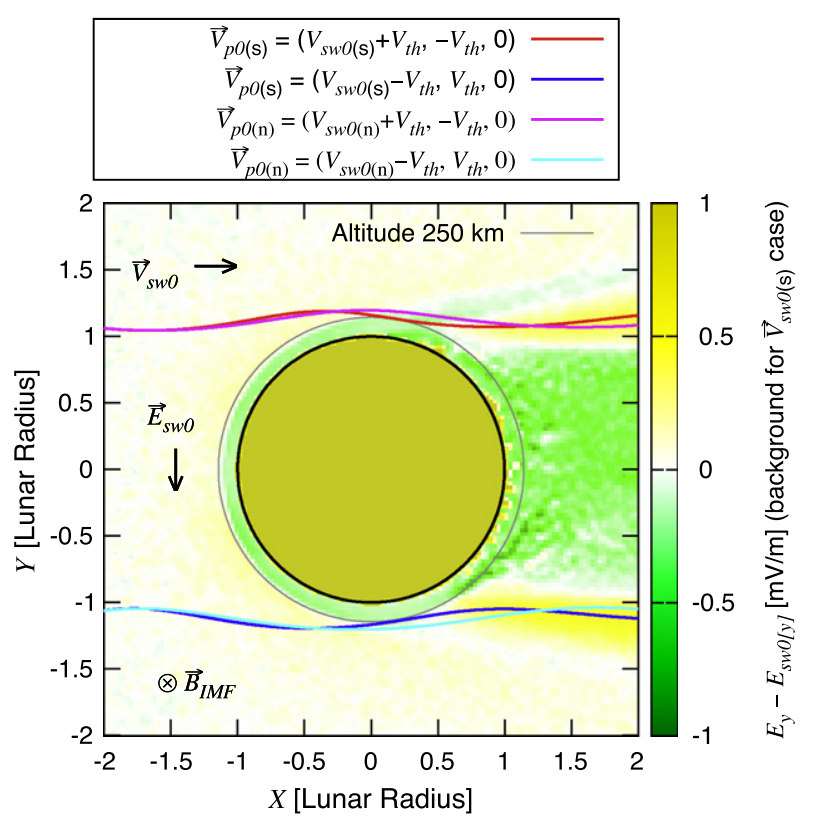

Fig. 12. The solar wind proton trajectories in the $x y$-plane for the slow $\left(V_{s w 0(s)}\right)$ and normal solar wind speed $\left(V_{s w 0(n)}\right)$ simulation cases. Note that although we only show the background $y$-component of the electric field $\left(E_{y}\right)$ distribution for the slow solar wind case, the trajectories for slow and normal solar wind cases are from different simulation results.

tion of the inward pointing electric field with a magnitude of $0.7 \mathrm{mV} / \mathrm{m}$ discussed in Nishino et al. (2009b). We did not observe such a deep entry as in the measurements.
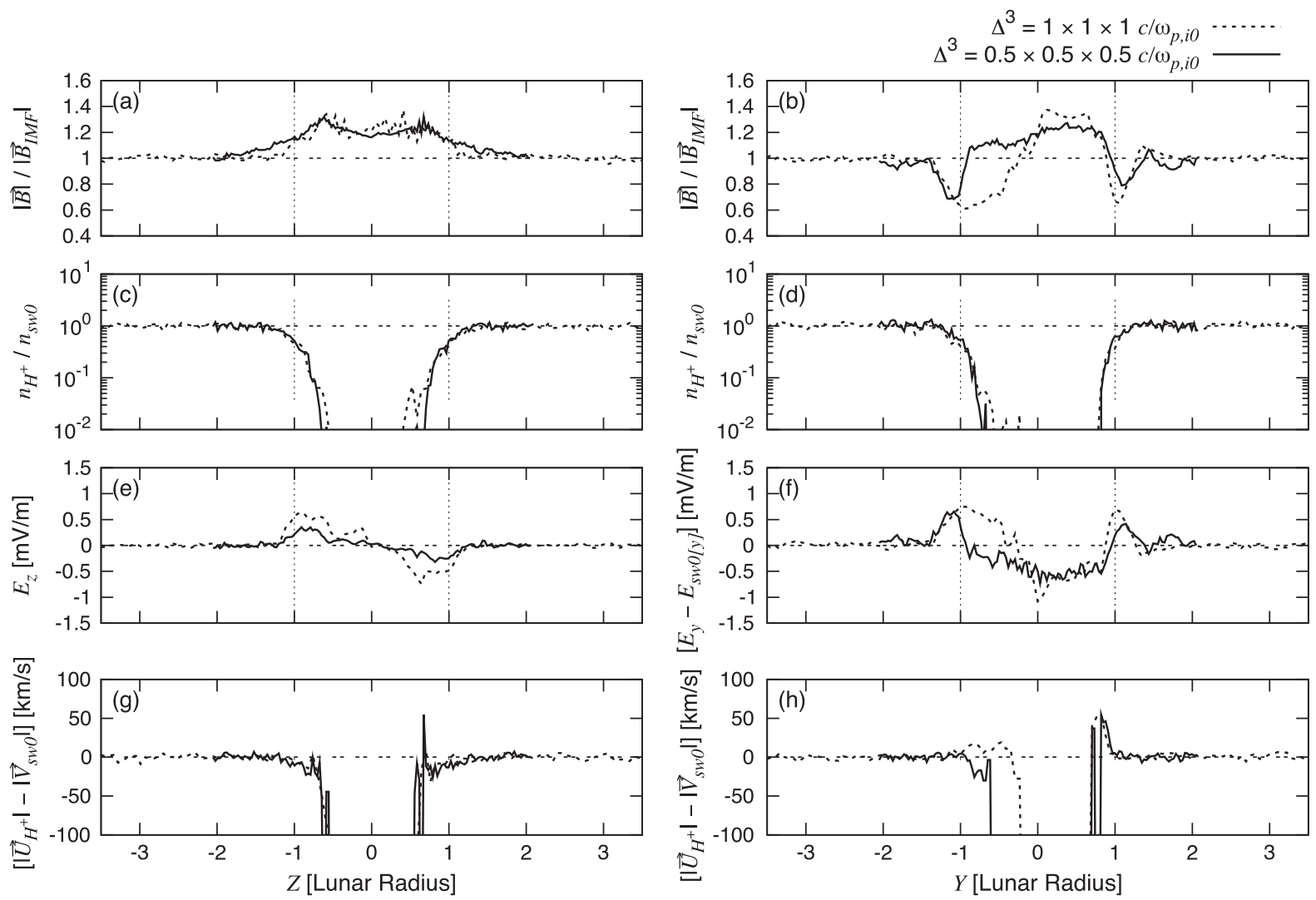

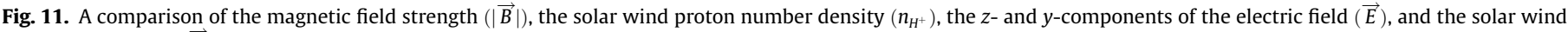
proton bulk speed $\left(\left|\vec{U}_{H^{+}}\right|\right)$distributions along the $z$ and $y$ directions at $X=2 R_{L}$ for two different grid size simulation cases. 
However, the resolution might still not be good enough to attempt to compare the results with the spacecraft measurements within only a few tens of kilometers from the surface (i.e., Kurata et al., 2005; Nishino et al., 2009b). The detailed electromagnetic field distribution near the surface region with the inclusion of magnetic remnants should be studied with better resolution on the surface. An adaptive hybrid simulation (Müller et al., 2011) is needed in future.

Note that the trajectories of the backscattered solar wind protons detected by the plasma instrument on the Kaguya spacecraft reach deep inside the wake region, with high speed and kinetic energy (Saito et al., 2008; Nishino et al., 2009a). The large-scale motion of this type of "self-pickup" solar wind protons ('Type-II entry' into the wake) is not significantly affected by the electric field at the wake boundary. However, it is expected that the Moon will be surrounded by a halo of neutral gas from the surface sputtering process, meaning that new pickup ions created near the wake could be subject to the electric field acceleration up and down the magnetic-field aligned direction. It is possible that the Artemis measurements of the lunar wake will provide important information on this novel effect.

\section{Summary}

We use a 3D hybrid simulation to study the electromagnetic field distribution in the lunar wake. From the analysis of the simulation results, we can conclude the following:

- With a conductivity of lower than $10^{-7} \mathrm{~S} / \mathrm{m}$, significant currents are not generated inside an object. When the IMF is measured as having almost free passage through the Moon, the lunar conductivity might be lower than $10^{-7} \mathrm{~S} / \mathrm{m}$.

- With a near-vacuum in ion density inside the wake, there is an observed increase in the strength of the magnetic fields by a factor of 1.4. As the magnetic field lines diffuse along with the ion motion, the magnetic field decreases in strength on both sides of the wake to create a cone shaped of depletion region. The Mach cone angle in the simulation is about $10^{\circ}$, which implies that the rarefaction wave is transported through fast-mode magnetosonic wave.

- Although the electric fields could not be generated by charge separation in the hybrid simulation, the convection of the ion motions and the magnetic fields could play a role. As the magnetic field lines bend toward the wake region, minor components of the magnetic fields can lead to the formation of an inward electric field along the field lines at the wake boundaries as in the measurements. Similarly, the field strength distribution can also induce electric fields perpendicular to the magnetic field lines.

- Due to fact that the pointing directions of the electric fields are perpendicular to the IMF at the wake boundaries, anisotropic flow of protons would occur, leading to asymmetrical distributions in both number density and magnetic field strength.

- If the IMF is not perpendicular to the solar wind flow directions, the inward diffusion along the magnetic field lines will differ at either end of the field lines. The ions will be accelerated at the "upstream" side and decelerated at the "downstream" ends.

The grid size used in the present work might not be good enough for a comparison of low altitude measurements. With larger influences from the magnetic remnants in the crust near the surface, better resolutions to deal with magnetic anomalies on the lunar surface will be needed. This can be done in future to acquire a better understanding of the electromagnetic field distribution near the vicinity of the Moon.

\section{Acknowledgments}

We thank the reviewers for their useful comments and suggestions. This work is supported in part by NSC Grant: NSC 96-2752M-008-011-PAE, the Ministry of Education under the Aim for the Top University Program NCU, and the Center for Computational Geophysics of the NCU with the CCG Contribution No.: NCUCCG99-0004. The work of J. Müller and U. Motschmann was supported by the Deutsche Forschungsgemeinschaft through Grant: M0539/16-1.

\section{References}

Bagdonat, T., 2004. Hybrid Simulation of Weak Comets. Ph.D. Thesis. Technische Universität, Braunschweig.

Bagdonat, T., Motschmann, U., 2002. From a weak to a strong comet-3d global hybrid simulation studies. Earth, Moon Planets 90, 305-321.

Birch, P.C., Chapman, S.C., 2001. Particle-in-cell simulations of the lunar wake with high phase space resolution. Geophys. Res. Lett. 28, 219-222.

Birch, P.C., Chapman, S.C., 2002. Two dimensional particle-in-cell simulations of the lunar wake. Phys. Plasmas 9, 1785-1789.

Bößwetter, A., Bagdonat, T., Motschmann, U., Sauer, K., 2004. Plasma boundaries at Mars: A 3-d simulation study. Ann. Geophys. 22, 4363-4379.

Clack, D., Kasper, J.C., Lazarus, A.J., Steinberg, J.T., Farrell, W.M., 2004. Wind observations of extreme ion temperature anisotropies in the lunar wake. Geophys. Res. Lett. 31, L06812.

Colburn, D.S., Currie, R.G., Mihalov, J.D., Sonett, C.P., 1967. Diamagnetic solar-wind cavity discovered behind Moon. Science 158, 1040-1042.

Farrell, W.M., Kaiser, M.L., Steinberg, J.T., Bale, S.D., 1998. A simple simulation of a plasma void: Applications to Wind observations of the lunar wake. J. Geophys. Res. 103, 23653-23660.

Farrell, W.M. et al., 2008. Loss of solar wind plasma neutrality and affect on surface potentials near the lunar terminator and shadowed polar regions. Geophys. Res. Lett. 35, L05105.

Glassmeier, K.H., 2000. Currents in Mercury's magnetosphere. In: Magnetospheric Current Systems. AGU Geophysical Monograph, vol. 118, pp. 371-380.

Halekas, J.S. et al., 2011. First results from ARTEMIS, a new two-spacecraft lunar mission: Counter-streaming plasma populations in the lunar wake. Space Sci. Rev. doi:10.1007/s11214-010-9738-8.

Halekas, J.S., Bale, S.D., Mitchell, D.L., Lin, R.P., 2005. Electrons and magnetic fields in the lunar plasma wake. J. Geophys. Res. 110, A07222.

Holmström, M., Fatemi, S., Futaana, Y., Nilsson, H., 2011. The interaction between the Moon and the solar wind. Earth, Planets Space, submitted for publication. arXiv:1104.1440 v1.

Holmström, M., Wieser, M., Barabash, S., Futaana, Y., Bhardwaj, A., 2010. Dynamics of solar wind protons reflected by the Moon. J. Geophys. Res. 115, A06206.

Janhunen, P., Kallio, E., 2004. Surface conductivity of Mercury provides current closure and may affect magnetospheric symmetry. Ann. Geophys. 22, 1829-1837.

Kallio, E., 2005. Formation of the lunar wake in quasi-neutral hybrid model. Geophys. Res. Lett. 32, L06107.

Kriegel, H., Simon, S., Müller, J., Motschmann, U., Saur, J., Glassmeier, K.H., 2009. The plasma interaction of Enceladus: 3d hybrid simulations and comparison with Cassini MAG data. Planet. Space Sci. 57, 2113-2122.

Kurata, M., Tsunakawa, H., Saito, Y., Shibuya, H., Matsushima, M., Shimizu, H., 2005 Mini-magnetosphere over the Reiner Gamma magnetic anomaly region on the Moon. Geophys. Res. Lett. 32, L24205.

Lipatov, A.S., Motschmann, U., Bagdonat, T., Grießmeier, J.M., 2005. The interaction of the stellar wind with an extrasolar planet-3d hybrid and drift-kinetic simulations. Planet. Space Sci. 53, 423-432.

Lyon, E.F., Bridge, H.S., Binsack, J.H., 1967. Explorer 35 plasma measurements in the vicinity of the Moon. J. Geophys. Res. 72, 6113-6117.

Michel, F.C., 1968. Magnetic field structure behind the Moon. J. Geophys. Res. 73, 1533-1542.

Müller, J., Simon, S., Motschmann, U., Schüle, J., Glassmeier, K.H., Pringle, G.J., 2011. A.I.K.E.F.: Adaptive hybrid model for space plasma simulations. Comput. Phys. Commun. 182, 946-966.

Ness, N.F., Behannon, K.W., Taylor, H.E., Whang, Y.C., 1968. Perturbations of the interplanetary magnetic field by the lunar wake. J. Geophys. Res. 73, 3421-3440.

Nishino, M.N. et al., 2009a. Solar-wind proton access deep into the near-Moon wake. Geophys. Res. Lett. 36, L16103.

Nishino, M.N. et al., 2009b. Pairwise energy gain-loss feature of solar wind protons in the near-Moon wake. Geophys. Res. Lett. 36, L12108.

Ogilvie, K.W. et al., 1996. Observations of the lunar plasma wake from the Wind spacecraft on December 27, 1994. Geophys. Res. Lett. 23, 1255-1258.

Owen, C.J., Lepping, R.P., Ogilvie, K.W., Slavin, J.A., Farrell, W.M., Byrnes, J.B., 1996. The lunar wake at $6.8 r_{l}$ : WIND magnetic field observations. Geophys. Res. Lett. 23, 1263-1266.

Roussos, E. et al., 2008. Plasma and fields in the wake of Rhea: 3-d hybrid simulation and comparison with Cassini data. Ann. Geophys. 26, 619-637.

Saito, Y. et al., 2008. Solar wind proton reflection at the lunar surface: Low energy ion measurement by MAP-PACE onboard SELENE (KAGUYA). Geophys. Res. Lett. 35, L24205. 
Samir, U., Wright, J.K.H., Stone, N.H., 1983. The expansion of a plasma into vacuum: Basic phenomena and processes and applications to space plasma physics. Rev. Geophys. Space Phys. 21, 1631-1646.

Schubert, G., Lichtenstein, B.R., 1974. Observations of Moon-plasma interactions by orbital and surface experiments. Rev. Geophys. Space Phys. 12, 592-626.

Simon, S., Bagdonat, T., Motschmann, U., Glassmeier, K.H., 2006a. Plasma environment of magnetized asteroids: A 3-d hybrid simulation study. Ann. Geophys. 24, 407-414

Simon, S., Bößwetter, A., Bagdonat, T., Motschmann, U., Glassmeier, K.H., 2006b. Plasma environment of Titan: A 3-d hybrid simulation study. Ann. Geophys. 24, 1113-1135.
Trávníček, P., Hellinger, P., Schriver, D., Bale, S.D., 2005. Structure of the lunar wake: Two-dimensional global hybrid simulations. Geophys. Res. Lett. 32 L06102.

Wang, Y.C., Müller, J., Motschmann, U., Ip, W.H., 2010. A hybrid simulation of Mercury's magnetosphere for the messenger encounters in year 2008. Icarus 209, 46-52.

Whang, Y.C., Ness, N.F., 1970. Observations and interpretation of the lunar Mach cone. J. Geophys. Res. 75, 6002-6010.

Wiehle, S. et al., 2011. First lunar wake passage of ARTEMIS: Discrimination of wake effects and solar wind fluctuations by $3 \mathrm{~d}$ hybrid simulations. Planet. Space Sci. 59, 661-671. 